\title{
LA COMISIÓN DE VENECIA Y LOS ESTADOS DE EMERGENCIA: LA NECESARIA PRESERVACIÓN DEL ESTADO DE DERECHO Y LA DEMOCRACIA CONSTITUCIONAL DURANTE LA CRISIS DE LA COVID-19 ${ }^{1}$
}

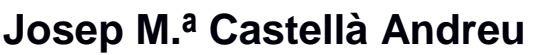

Catedrático de Derecho Constitucional de la Universidad de Barcelona Miembro por España de la Comisión de Venecia del Consejo de Europa

Cómo citar este artículo / Citation: Castellà Andreu, J.M. (2020). La Comisión de Venecia y los estados de emergencia: la necesaria preservación del Estado de Derecho y la democracia constitucional durante la crisis de la covid-19. Biglino Campos, P.; Durán Alba, F. Los Efectos Horizontales de la COVID sobre el sistema constitucional, Colección Obras colectivas, Fundación Manuel Giménez Abad, Zaragoza. DOI: https://doi.org/10.47919/FMGA.OC20.1019

SUMARIO: I. INTRODUCCIÓN - II. COMISIÓN DE VENECIA Y ESTADOS DE EMERGENCIA: TIPOS DE ESTADOS EXCEPCIONALES Y PRINCIPIOS QUE LOS RIGEN - 1. La opción por el modelo del Estado de Derecho - 2. Tipos de estados de emergencia y su regulación - 3. Principios que rigen los estados de emergencia - III. CRITERIOS DE LA COMISIÓN DE VENECIA EN RELACIÓN CON LA AFECTACIÓN A LOS DERECHOS Y A LA SEPARACIÓN DE PODERES DURANTE LOS ESTADOS DE EMERGENCIA - 1. Limitación y suspensión de derechos fundamentales - 2. Especial atención al derecho de sufragio y la celebración de elecciones - 3 . Redistribución de los poderes durante la emergencia - 4. Control judicial y mantenimiento del Estado de Derecho - IV. CONCLUSIÓN

\footnotetext{
${ }^{1}$ Este trabajo se enmarca en el proyecto EU H2020, Democratic Efficacy and the Varieties of Populism in Europe (Demos), Grant No 822590, en el proyecto financiado por el Ministerio de Ciencia e Innovación PID2019-104414GB-C32, "Instrumentos contramayoritarios en el Estado constitucional" y en las actividades del Grupo de Estudios sobre la Democracia y el Constitucionalismo (GEDECO), grupo consolidado por la Generalitat de Cataluña 2017 (SGR 1595). Se desarrollan aquí las reflexiones iniciadas en la Presentación de "Compilación de la Comisión de Venecia de opiniones e informes sobre Estados de emergencia", RGDC, núm. 32, 2020.
} 


\section{INTRODUCCIÓN}

La crisis sanitaria ocasionada por el virus covid-19 es una emergencia de alcance mundial, aunque las respuestas jurídicas a la misma sean eminentemente nacionales. La pandemia ha suscitado en España, como en los demás países afectados, reflexiones jurídico-constitucionales sobre la forma de afrontarla por parte de las autoridades públicas, sobre los problemas de la aplicación de los estados excepcionales que los ordenamientos jurídicos suelen prever a la crisis actual y sobre la afectación de las medidas adoptadas a los derechos fundamentales y la división de poderes, constitucionalmente establecidos. Todo ello plantea discusiones y preocupaciones políticas y jurídicas similares, a pesar de las particularidades de cada Estado a la hora de afrontar la crisis. En el fondo late la tensión, en momentos de anormalidad política, social y económica, entre necesidad de respuestas eficaces y rápidas a la pandemia y el imprescindible mantenimiento del Estado de Derecho y de la democracia constitucional, de modo particular, los frenos y contrapesos entre poderes y la vigencia de los derechos fundamentales. Dichos momentos excepcionales, de origen y naturaleza muy diferente, por cierto, son, cada vez más, periodos que se prolongan en el tiempo y convierten la excepción en lo ordinario (11-S de 2001, o la crisis financiera de 2008, la crisis secesionista catalana de 2012 en España). La respuesta de los ordenamientos a tales crisis no se ha circunscrito a la aplicación de los estados excepcionales constitucionalmente previstos, que ha sido más bien extraordinaria, sino que se han utilizado en su lugar instrumentos normativos de crisis más amplios y de distinto tipo. Hay, no obstante, ciertos rasgos que se repiten: el protagonismo de los gobiernos y el uso de los decretos ley, la restricción de la libertad en nombre de la seguridad, o la intervención de los poderes públicos en ámbitos crecientes de la vida social o económica, cada vez más aceptadas por la opinión pública. El debate asume en los últimos tiempos particular relevancia ante la por lo menos discutible utilización de poderes excepcionales por parte de algunos gobiernos, entre ellos el español, para afrontar la crisis sanitaria y las consecuencias sociales y económicas de la misma.

Constitucionalistas y administrativistas han polemizado, en España y en muchos otros países, sobre el alcance de las medidas restrictivas y suspensivas de derechos, sobre la autoridad competente para decidirlas y 
sobre la efectividad de los controles políticos y jurisdiccionales. Sobre todo, ello incide, además, la opción concreta por el tipo de legislación de emergencia a aplicar, de las varias previstas, a la presente pandemia en los distintos países. La opción por una u otra vía jurídica que han decidido los respectivos gobiernos, con participación, de un tipo o de otro, del Parlamento, no es un hecho inocuo, de ahí la controversia política y jurídica suscitada.

Así pues, dicha crisis plantea problemas comunes de alcance supranacional y también respuestas que trascienden el ámbito de cada Estado. Entre ellos, nos vamos a detener, a continuación, en: 1) los problemas constitucionales generados por un tipo de crisis para la que los ordenamientos no suelen estar suficientemente preparados, puesto que el Derecho de excepción está diseñado principalmente para prevenir o afrontar crisis que afectan ya sea al orden público y la seguridad del Estado, ya a catástrofes naturales o humanas acotadas en el espacio y el tiempo, y 2) las respuestas jurídico-constitucionales adecuadas para afrontar una crisis de la magnitud y duración de la presente.

Para aproximarnos a las situaciones de emergencia son posibles varias perspectivas jurídicas: la del Derecho constitucional de cada Estado, la del Derecho internacional, la del Derecho constitucional comparado y la del llamado Derecho transnacional. La Comisión Europea para la democracia por el Derecho, del Consejo de Europa, conocida como Comisión de Venecia, está entre los organismos internacionales que han intervenido en relación con la presente crisis sanitaria global.

La Comisión de Venecia ha sido incluida por el profesor Paul Craig dentro de los Ilamados Órdenes Legales Transnacionales (OLT), y lleva a cabo un papel transnacional importante en el constitucionalismo global, por su función, miembros y valores, puesto que opera sobre normas jurídicas relativas a la democracia, los derechos humanos y el Estado de Derecho, en definitiva, en relación con el constitucionalismo ${ }^{2}$.

\footnotetext{
${ }^{2}$ Paul CRAIG, "Constitucionalismo transnacional: la contribución de la Comisión de Venecia", Teoría y Realidad Constitucional, núm. 40, 2017, pág. 90 ss. El autor adopta las definiciones de Halliday y Shaffer y las aplica a la Comisión de Venecia.
} 
La Comisión de Venecia ha intervenido desde el comienzo de la covid-19 con la publicación de tres documentos hasta ahora ${ }^{3}$ : primero, el 16 de abril de 2020 la Compilación de la Comisión de Venecia Opiniones e informes sobre los estados de emergencia (CDL-PI(2020)003) ${ }^{4}$. Segundo, el 26 de mayo de 2020 las reflexiones de algunos de sus miembros más ilustres: "Respeto por la democracia, derechos humanos y el Estado de Derecho durante los estados de emergencia - Reflexiones", documento que fue "tomado en consideración" por el Pleno de la Comisión de junio de este año (CDL-PI(2020)005rev) ${ }^{5}$. Tercero, la adopción por el Pleno de la sesión de 8 y 9 de octubre de 2020 de un Informe provisional (Interim Report) sobre las medidas tomadas por los Estados de la Unión Europea como resultado de la crisis covid-19 y su impacto en la democracia, el Estado de Derecho y los derechos fundamentales (CDL$A D(2020) 018)$, informe que había solicitado el Presidente del Parlamento Europeo $^{6}$. Este último documento ya alude expresamente a la pandemia actual desde el título y el segundo la menciona en el cuerpo del mismo. Ahora bien, el análisis de ambos documentos no tiene en cuenta la segunda ola de la pandemia, iniciada en septiembre-octubre 2020, en la que estamos inmersos cuando se escriben estas líneas, con características en parte distintas a la primera ola, tanto desde el punto de vista fáctico, o de la incidencia de la pandemia, como del jurídico-político, o respuesta a la misma. Cada uno de los textos presenta diferencias tanto de forma o tipo de documento de que se trata, como de estructura y contenido, aunque obviamente haya aspectos comunes y reiterados en todos ellos: la atención prestada a la afectación de la emergencia a la división de poderes y los derechos así como a las elecciones (ámbitos propios de la actuación de la Comisión), y los criterios que han de regir para la declaración del estado de emergencia y su duración así como para la adopción de las medidas necesarias. Asimismo, el secretariado de la Comisión ha creado un Observatorio sobre los estados de emergencia, mediante la recopilación y publicación en su página web de los resultados de un cuestionario sobre el estado de la cuestión en los 62 Estados miembros

\footnotetext{
${ }^{3}$ Existe traducción al español de los tres textos, aunque no tiene carácter oficial: todos los documentos de la Comisión de Venecia están redactados en las lenguas oficiales de la misma, el inglés y el francés.

${ }^{4}$ https://www.venice.coe.int/webforms/documents/default.aspx?pdffile=CDL-PI(2020)003-spa

${ }^{5}$ Autores: Nicos Alivizatos, Veronika Bílková, Ian Cameron, Oliver Kast y Kaarlo Tuori. Ver: https://www.venice.coe.int/webforms/documents/default.aspx?pdffile=CDL-PI(2020)005rev-spa

${ }^{6}$ Ponentes: Nicos Alivizatos, Veronika Bílková, Oliver Kast, Rafael Rubio, Kaarlo Tuori y Ben Vermeulen. Ver:

https://www.venice.coe.int/webforms/documents/default.aspx?pdffile=CDL-AD(2020)018-e 
(previsiones constitucionales y legislativas, así como su aplicación), que han contestado los miembros del Comisión de cada Estado. Dado que la pandemia sigue y los Estados siguen aplicando un derecho de emergencia, la Comisión de Venecia continuará con su seguimiento y evaluación de las reacciones de los Estados miembros del Consejo de Europa a esta situación de emergencia.

Con estos documentos, la Comisión de Venecia asume la relevancia de la actual crisis sanitaria en nuestras sociedades y su incidencia sobre los objetivos fundacionales del Consejo de Europa y de la misma Comisión de Venecia desde su origen en 1990, ahora hace treinta años: la salvaguarda de la democracia, los derechos humanos y el Estado de Derecho. Ello justifica, de entrada, la atención a la emergencia derivada de la covid-19. Creo, además, que hay otra razón implícita de la atención prestada a la actual crisis por parte de la Comisión de Venecia: las pautas que había ofrecido con anterioridad a situaciones de emergencia estaban pensadas, igual que la del derecho de excepción de los ordenamientos constitucionales, para hacer frente ya sea graves problemas de orden público y crisis de seguridad nacional, ya sea catástrofes naturales o sanitarias de menor alcance. La primera novedad de la presente crisis sanitaria, y sus efectos sobre la economía, está en su gravedad, duración y efectos. Aunque muchos de los principios generales del derecho de emergencia sean aplicables a la nueva situación, era necesaria una puesta al día y una adaptación. A ello contribuyen los documentos segundo y tercero -las Reflexiones y el Informe provisional-, mientras que el primero, la Compilación, es un recordatorio de la doctrina reiterada de la Comisión de Venecia sobre el derecho de emergencia. Por último, el Informe provisional se centra en las actuaciones llevadas a cabo por los Estados de la UE (y el Reino Unido) para hacer frente a la pandemia y a sus efectos sobre la democracia, el Estado de Derecho y los derechos humanos (hasta octubre de 2020, fecha de su aprobación).

Antes de proceder al análisis de los contenidos de los documentos y las conclusiones de la Comisión de Venecia acerca de la emergencia, conviene aclarar los tipos de documentos emitidos por la Comisión que han sido aludidos. Los dos primeros son calificados como "estudios". Las "compilaciones" son documentos preparados por el Secretariado de la Comisión de Venecia, con sede en Estrasburgo, que recopilan los fragmentos más destacados de informes y opiniones existentes sobre un determinado asunto. Las 
compilaciones no innovan la doctrina del órgano consultivo del Consejo de Europa y son periódicamente (re)elaboradas para permitir a los miembros, juristas y público interesado una primera aproximación general a lo que la Comisión ya ha ido señalando en documentos aprobados en momentos distintos, a partir de casos y circunstancias particulares ${ }^{7}$. La técnica de elaboración de la Compilación explica el carácter no siempre coincidente y a veces reiterativo del texto. Las "Reflexiones" citadas son un estudio doctrinal elaborado ex novo, de ahí su mayor coherencia en su contenido, por algunos miembros o expertos y ofrecen pautas orientativas sobre un asunto. Ambos tipos de "estudios" pueden ayudar a los miembros y analistas en el estudio de una materia determinada. La Comisión "asume" (endorse) las compilaciones y "toma en consideración" (take into account) las reflexiones. En definitiva, las hace suyas, pero ninguna de las dos se corresponde con los documentos oficiales "que aprueba" el Pleno de la Comisión: informes y opiniones. De hecho, tienen identificación diferente: CDL-PI en el caso de compilaciones $u$ otros documentos informativos, CDL-AD, para los informes y opiniones aprobados por el Pleno de la Comisión y que contienen la doctrina oficial de la misma. Este es el caso del tercer documento objeto de análisis, el "Informe provisional" (Interim Report) sobre las medidas adoptadas por los Estados miembros de la UE como resultado de la crisis de la covid-19, de octubre de 2020. Los informes (report) tratan un determinado asunto, con carácter general, y son realizados normalmente ya sea a iniciativa de la propia Comisión ya de organismos como la Asamblea Parlamentaria del Consejo de Europa o, en el caso en estudio, del Parlamento Europeo ${ }^{8}$. En cambio, las opiniones (opinion) versan sobre la adecuación de proyectos normativos a estándares internacionales, aprobados, por lo general, a instancia de las autoridades de los Estados parte de la Comisión de Venecia, además de otros legitimados, como

\footnotetext{
${ }^{7}$ En el caso que nos ocupa, la Compilación recoge extractos relevantes de las opiniones e informes previamente adoptados por la Comisión de Venecia relacionados con los estados de emergencia. Por un lado, informes generales, en particular, el dedicado a los Emergency powers (CDL-STD(1995)012), además de otros que afectan a aspectos sectoriales del tema, como los recientes Rule of Law checklist o Criterios de verificación del Estado de Derecho de 2016 (CDL-AD(2016)007), y de los Parámetros de la relación entre mayoría parlamentaria y oposición en una democracia de 2019 (Parameters on the Relationship between the Parliamentary Majority and the Opposition in a Democracy: a checklist, CDL-AD(2019)015). Por otro, de opiniones en relación con casos concretos surgidos a raíz de reformas constitucionales y legales en Estados miembros de la Comisión (como las opiniones sobre Armenia de 2015, Francia de 2016, Túnez de 2013 y Turquía de 2016 y 2017, entre las más recientes y a las que se irá aludiendo a lo largo del trabajo).

${ }^{8}$ El carácter "interim" del Informe significa que puede ser completado ulteriormente.
} 
la citada Asamblea Parlamentaria. Las opiniones y los informes son debatidos y aprobados por el Pleno de la Comisión de Venecia, a partir del proyecto elaborado por unos relatores, habitualmente miembros de la Comisión, y con el apoyo eficaz de los juristas del Secretariado.

La Comisión de Venecia tiene en cuenta, como parámetros para redactar sus documentos, en primer lugar, el Derecho internacional público (en el presente caso se citan especialmente el Pacto Internacional de Derechos Civiles y Políticos, la Convención Americana de Derechos Humanos y la Convención Europea de Derechos Humanos, además de algunas sentencias del TEDH), y, en segundo lugar, aportaciones relevantes del Derecho constitucional comparado sobre la materia. El Informe aprobado en octubre de 2020 se centra en los Estados de la Unión Europea y el Reino Unido, mientras que los dos anteriores aluden también a los demás Estados del Consejo de Europa.

Estamos ante aportaciones de la Comisión de Venecia útiles, que nos pueden servir como parámetros orientativos para valorar el uso de los poderes excepcionales que están llevando a cabo los diferentes Estados durante la pandemia de la covid-19 y, en particular, en el caso español. A continuación, vamos a resumir y comentar los documentos referidos, siguiendo la sistemática siguiente: primero, los tipos de estados de emergencia y los principios que deben guiar su regulación y aplicación; y segundo, los criterios de la Comisión de Venecia en relación con la suspensión y restricción de derechos, con particular atención a la afectación al derecho de sufragio de la celebración o aplazamiento de las elecciones en tiempos de emergencia, así como respecto a la redistribución de poderes durante la vigencia de los estados excepcionales y al control parlamentario y judicial de las decisiones gubernamentales.

\section{COMISIÓN DE VENECIA Y ESTADOS DE EMERGENCIA: TIPOS DE ESTADOS EXCEPCIONALES Y PRINCIPIOS QUE LOS RIGEN}

\section{La opción por el modelo del Estado de Derecho}

Como recuerdan las Reflexiones, caben teóricamente dos aproximaciones a las situaciones de anormalidad o excepción por parte de los Estados: primera, la denominada "soberana", que se corresponde con la decisionista y que evoca la 
forma de abordarlas de Carl Schmitt, basada en la búsqueda por la autoridad de la salud pública sin el límite de lo previsto por el ordenamiento o bien forzando su interpretación en tal dirección. Y segunda, la propia del Estado de Derecho, en la que el Derecho, y la Constitución en particular, regula y, por ello, racionaliza y limita el ejercicio del poder en los estados excepcionales, aunque sea haciéndolo de modo distinto a las situaciones de normalidad. La Comisión de Venecia opta, como no podía ser de otro modo, por la segunda aproximación ${ }^{9}$, como corresponde hacerlo al Estado constitucional. A continuación, veremos los efectos de la previsión por el ordenamiento jurídico de los estados de emergencia.

\section{Tipos de estados de emergencia y su regulación}

Adviértase, de entrada, que "estado de emergencia" es el término genérico que suele utilizar la Comisión de Venecia para englobar los distintos estados excepcionales, que reciben nombres diferentes en cada ordenamiento jurídico, además de otras regulaciones de emergencias. Por ello, la Comisión parece que opta por un concepto amplio y material de las emergencias, frente al más estricto de los estados excepcionales, formalmente identificados en la Constitución (como ocurre en España).

La Comisión de Venecia distingue los diferentes tipos de estados de emergencia que suelen preverse por los ordenamientos estatales, cuando se regula más de uno genérico, normalmente siguiendo un criterio objetivo -o del tipo de emergencia de que se trate- $y / u$ otro referido a la gravedad de la situación extraordinaria ${ }^{10}$. En consecuencia, para su distinción se deberá tener en cuenta la "naturaleza, la severidad y la duración de la situación extraordinaria". Dichos aspectos determinarán el "tipo, extensión y duración de las medidas de emergencia" ${ }^{11}$. Al introducir esta pluralidad de criterios de identificación de cada tipo de estado de emergencia se opta por un planteamiento flexible que, de entrada, asume la pluralidad de aproximaciones en Derecho comparado, y que, además, resulta oportuno para permitir cierta modulación en la aplicación del estado de emergencia, en función de la

\footnotetext{
${ }^{9}$ Reflexiones...CDL-PI(2020)005, párr. 8; Interim Report...CDL-AD(2020)018, párr. 18 y 19.

${ }^{10}$ Emergency Powers, CDL-STD(1995)012, en la Compilación, pág. 6. La cita de las páginas es a la de la Compilación en su versión original inglesa; cuando se citan párrafos, en cambio, se corresponden con los del informe u opinión originarios.

${ }^{11}$ Interim Report...CDL-AD(2020)018, párr. 20.
} 
gravedad y duración del supuesto de hecho concreto. Pero, al mismo tiempo, tal mixtificación de criterios en la delimitación de los distintos estados de emergencia puede dar lugar a que la autoridad competente de la declaración de tal estado opte por utilizar el tipo de estado menos garantista y/o que mayor intervención gubernativa posibilite. De ahí la necesidad, como se verá, de precisar al máximo las causas que determinan la aplicación de los distintos estados de emergencia.

Precisamente la decantación por un criterio objetivo por parte de la Ley orgánica reguladora de los estados excepcionales, esto es, de alarma, excepción y sitio (LO 4/1981), a partir de la previsión indeterminada de los supuestos de aplicación de los mismos en el art. 116 CE, llevó en España, a mi juicio acertadamente, a declarar el estado de alarma en marzo de 2020, y no el de excepción, porque el supuesto de hecho se adecuaba al previsto en el art. 4.b) de la Ley orgánica ("crisis sanitarias, tales como epidemias y situaciones de contaminación graves"). Pero la intensidad de las restricciones a los derechos que conllevaba generó un debate doctrinal sobre el encaje de las medidas adoptadas a la regulación del estado de alarma, en el que algunos autores defendieron con argumentos poderosos que nos encontrábamos ante un supuesto de estado de excepción, que requería las garantías de este ${ }^{12}$.

La Comisión de Venecia en el Interim Report de octubre 2020 ofrece un cuadro general de la situación en los Estados de la UE y el Reino Unido, en que clasifica las respuestas jurídicas de dichos Estados, según hayan declarado o no el estado emergencia y según la norma de cobertura de las medidas de emergencia adoptadas. A ojos españoles llama la atención que se utilice un criterio amplio de estado de emergencia, que incluye tanto los estados excepcionales previstos constitucionalmente (criterio estricto de estados excepcionales, seguido en nuestro país) como otras regulaciones de emergencia sectorial, como es la sanitaria, reguladas por la legislación correspondiente. Dentro del primer supuesto, de declaración de estados de emergencia, unos Estados han optado por la utilización de alguno de los estados excepcionales constitucionalmente previstos (nueve países, siete de

\footnotetext{
${ }^{12}$ Por todos Manuel Aragón Reyes, cfr. "Editorial. Covid-19: aproximación constitucional a una crisis", Revista General de Derecho Constitucional, núm. 32, 2020. En varios post o intervenciones en los medios de comunicación vertieron sus opiniones al respecto, entre otros, J. Díaz Revorio, C. Flores Juverías y C. Vidal. En contra E. Vírgala, J. Tajadura J. De Miguel y J.M. Castellà.
} 
ellos por decisión gubernamental, entre ellos España, y otros dos del Parlamento, entre ellos Portugal); y otros han preferido no aplicar dichos estados excepcionales (aun cuando estuviesen contemplados en sus constituciones respectivas) y acogerse a la legislación prevista para emergencias sanitarias, con la adopción ex profeso de medidas bien por el Parlamento bien por el Gobierno (cinco países, entre ellos Alemania, Francia e Italia). Por el contrario, otros catorce Estados han preferido utilizar la legislación ordinaria, con adaptaciones a las circunstancias, sin declarar estados excepcionales ni recurrir a legislación de emergencia (así Austria, Bélgica, Grecia o el Reino Unido) ${ }^{13}$. Así pues, la Comisión, en el Informe de octubre, lleva a cabo un análisis siguiendo un criterio material o sustantivo, sin que se limite a estudiar solo los casos de los países que han procedido a una declaración jurídica de los poderes de emergencia. De este modo, toma en consideración y valora la respuesta jurídica a la pandemia de todos los Estados de la UE y el Reino Unido, independientemente de la vía elegida para afrontar la emergencia sanitaria.

A lo anterior cabría añadir que las respuestas dadas por los Estados no han sido estáticas, sino que ha ido variando a medida que pasaba el tiempo, cambiaban las circunstancias o, simplemente, el Gobierno prefería tal cambio. El caso español es un buen ejemplo de ello. Podemos distinguir al menos tres fases distintas y sucesivas hasta la fecha (marzo-noviembre 2020). Primera, tras un primer momento de toma de medidas descoordinadas y en buena medida improvisadas por parte de los distintos poderes públicos, nacionales y territoriales, el Gobierno del Estado decretó el estado de alarma, prorrogado por el Congreso hasta en seis ocasiones por periodos de 15 días (Real Decreto 463/2020, vigente desde el 14 de marzo al 21 de junio 2020). Segunda, tras el cese del estado de alarma, se optó por el modelo, ensayado ya en las últimas prórrogas del mismo, conocido como de "cogobernanza", consistente en la recuperación de competencias de dirección política por parte de las Comunidades Autónomas y cierta coordinación por parte del Gobierno central y cooperación interterritorial a través del Consejo Interterritorial de Salud. No entramos aquí a valorar su funcionamiento, con polémica política y jurídica entre el Gobierno central y algunas CCAA, como la Comunidad de Madrid. En esta segunda fase tuvo lugar la aprobación de regulaciones autonómicas muy

\footnotetext{
${ }^{13}$ Interim Report...CDL-AD(2020)018, párr. 35-38 y 41-43.
} 
variadas bajo la cobertura forzada de la Ley orgánica 3/1986, de 14 de abril, de salud pública (art. 3), y de la Ley 29/1998, de la jurisdicción contenciosoadministrativa (recientemente modificada en relación con los tribunales responsables de autorizar o ratificar las medidas administrativas adoptadas por las distintas autoridades sanitarias en relación con las medidas urgentes y necesarias para la salud pública que supongan limitación no individualizada de derechos fundamentales, Ley 3/2020, de 18 de setiembre, arts. 8,10 y 11). Y tercera fase, coincidiendo con el repunte de la pandemia en la conocida como segunda ola y los problemas legales ocasionados por la aplicación de la normativa ordinaria citada, con la utilización de nuevo del estado de alarma, primero para algunos municipios de la Comunidad de Madrid (Real Decreto 900/2020, de 9 de octubre) y después para todo el Estado (Real Decreto 926/2020, de 25 de octubre). Este decreto, con prórroga aprobada por el Congreso para seis meses (Decreto 956/2020, de 3 de noviembre), habilita a las autoridades autonómicas para la adopción de medidas restrictivas de la movilidad y de reuniones sociales, sin tener que solicitar autorización judicial. No se entra aquí a comentar su significado y peligros, aunque luego se aludirá a alguno de ellos.

Una vez realizado el mapping de la situación en los países de la UE, la Comisión de Venecia introduce un criterio prescriptivo en relación con el uso debido por los Estados de los poderes de emergencia. En este punto, se muestra partidaria del "sistema constitucional" o "estado de emergencia de iure" o "de estado constitucional de iure de poderes de emergencia" (por aludir a las formas de denominarlo), porque ofrece mayores garantías a los derechos fundamentales, el Estado de Derecho y la democracia. Este sistema da mayor seguridad jurídica que "el sistema extraconstitucional" o "estado de emergencia de facto" 14 . Sin embargo, la Comisión, como ya hemos referido, deja a la libre elección de los Estados la opción por una legislación de emergencia basada en los estados excepcionales constitucionalizados 0 en otras previsiones legislativas. Esta consideración amplia del modelo o tipo denominado "constitucional" puede inducir a confusión por el nombre que lo designa, ya que permite justificar la opción por estados de emergencia derivados de principios constitucionales no escritos o incluidos en una "ley (orgánica)" (sic) basada en la Constitución. En realidad, el tipo que ofrece mayores garantías es el que se

${ }^{14}$ Interim Report...CDL-AD(2020)018, párr. 29-31, 40 y 57; Reflexiones...CDL-PI(2020)005, párr. 22-24. 
deriva de la Constitución misma en sentido estricto, esto es, el primer supuesto del primer tipo o modelo descrito más arriba. Así, la interpretación amplia del criterio constitucional o jurídico, no empece que identifique la norma constitucional como la más adecuada para establecer las "previsiones básicas", lo que incluye la identificación de dichos estados y la delimitación de los mismos, dado que los poderes de emergencia "habitualmente restringen principios constitucionales básicos" ${ }^{15}$. Otra cosa es que el desarrollo corresponda al legislador, y ahí la Comisión muestra una preferencia porque sea un legislador cualificado, como el orgánico. Dicha regulación ha de ser general y aprobada con carácter previo a la declaración, durante tiempos de normalidad, según subrayan las Reflexiones ${ }^{16}$.

\section{Principios que rigen los estados de emergencia}

Los estados excepcionales en sentido amplio, o de emergencia, tal y como los aborda la Comisión de Venecia, deben ser regidos por distintos principios que operan, con las debidas adaptaciones, en tres momentos y situaciones distintos: su regulación, activación y aplicación ${ }^{17}$. Tales principios orientan los ámbitos fundamentales de la vida pública y privada sobre los que se proyecta el derecho de emergencia: los derechos fundamentales y la división de poderes. La Comisión de Venecia presta, asimismo, especial atención a la aplicación de los principios al ejercicio del derecho de sufragio y la celebración de elecciones.

Los principios a tener en cuenta se van desgranando en los distintos documentos, sin que se citen todos ellos de forma sistemática en un único elenco cerrado y exhaustivo. Unas veces se hace referencia a principios generales (como el de legalidad o proporcionalidad), dentro de los cuales cabe incluir otros más particulares, otras veces se opta, en cambio, por elencos más minuciosos y detallados. Además, los principios están relacionados entre sí y se producen solapamientos entre sus contenidos. Esta diferente técnica de relación de los principios es consecuencia de las diferentes autorías de los distintos documentos y del origen de los mismos en situaciones particulares y momentos variados. Por ello la enumeración que llevamos a cabo a

\footnotetext{
${ }^{15}$ Reflexiones...CDL-PI(2020)005, párr. 26; Emergency Powers, CDL-STD(1995)012, en la Compilación, pág. 30.

${ }^{16}$ Reflexiones...CDL-PI(2020)005, párr. 29 y 30.

${ }^{17}$ La diferencia entre los dos últimos en Interim Report...CDL-AD(2020)018, párr. 28. 
continuación supone, por nuestra parte, un intento de ordenación lo más amplia posible de los mismos, admitiendo posibles reiteraciones.

Así, podemos destacar los principios de legalidad o de Estado de Derecho, de necesidad y de proporcionalidad en sentido estricto ${ }^{18}$. A ellos hay que sumar otros como el de carácter expreso de su proclamación, y el de excepcionalidad $^{19}$, así como el principio de diferenciación entre los varios estados excepcionales, según el cual no puede confundirse ni tratarse por igual toda emergencia (nos hemos referido al mismo en el apartado anterior) ${ }^{20}$. Las Reflexiones, a modo recapitulatorio, mencionan, además de los citados principios de Estado de derecho, necesidad y proporcionalidad, el de temporalidad, el de efectivo control o escrutinio parlamentario y judicial, el de predictibilidad de la legislación de emergencia y el de leal cooperación entre instituciones $^{21}$.

Antes de referirnos, en los apartados siguientes, más detenidamente a la aplicación de tales principios a la limitación de derechos y a la organización de los poderes, conviene aludir, con carácter general y previo, al contenido y ciertas consecuencias derivadas de algunos de los principios mencionados.

La observancia del principio de legalidad o Estado de Derecho, y en concreto, el de la seguridad jurídica, conlleva para la Comisión de Venecia, entre otras consecuencias, que las normas reguladoras del estado de emergencia sean: a) previas a la declaración del estado de emergencia; b) sean claras y se eviten las cláusulas abiertas ("la regulación de estas facultades debe ser lo más detallada posible") ${ }^{22}$; y c) cuando el Parlamento delegue en el Ejecutivo "los objetivos, el contenido y el alcance de esa delegación de poderes deben definirse explícitamente en un acto legislativo." ${ }^{23}$, cuestión sobre la que se volverá más tarde. Así, se evita que se dejen en manos de los poderes ejecutivos amplias esferas de actuación sin habilitación clara y determinada.

\footnotetext{
${ }^{18}$ Emergency Powers, CDL-STD(1995)012, en la Compilación, pág. 5.

${ }^{19}$ CDL-AD(2016)006, Opinion on the Draft Constitutional Law on "Protection of the Nation" of France, párrafo 28, en la Compilación , pág. 7.

${ }^{20} \mathrm{CDL}-\mathrm{AD}(2017) 005$, Turkey - Opinion on the amendments to the Constitution adopted by the Grand National Assembly on 21 January 2017 and to be submitted to a National Referendum on 16 April 2017, párr. 73, en la Compilación, pág. 8.

${ }^{21}$ Reflexiones...CDL-PI(2020)005, párr. 6-16.

22 Interim Report...CDL-AD(2020)018, párr. 58.

${ }^{23}$ Interim Report...CDL-AD(2020)018, párr. 58. Con cita del CDL-AD(2016)007, Rule of Law Checklist, párr. 1.4.iii.
} 
En relación con el principio de temporalidad, la Comisión de Venecia constata, por un lado, que las medidas excepcionales con las que la mayoría de los Estados europeos han afrontado la pandemia se dictan para un periodo determinado, sometido a prórroga. Solo en unos pocos Estados se aprobaron sin fijar un límite temporal, sino utilizando cláusulas relativas a la permanencia de la situación de base (Croacia y Hungría). En tales casos, la Comisión recomienda que, para considerar tales declaraciones conforme a derecho, "haya control con regularidad de la situación" 24 . Por otro lado, como criterio prescriptivo, se indica que, sea cual sea el modo empleado de regulación, además del sometimiento a control -parlamentario y judicial-, debe cesar la vigencia de las medidas tan pronto acaben las circunstancias que propiciaron su aprobación (así, por ejemplo, en los cambios en la distribución de poderes) y "volver a la normalidad" 25 . Sobre este último punto, la Comisión admite, sin embargo, que, en primer lugar, puedan flexibilizarse las medidas de emergencia según la evolución de la situación y, en segundo lugar, dada la prolongación en el tiempo de determinados efectos de la situación causante de la emergencia, tras el cese del estado de emergencia, pueda mantenerse un régimen jurídico aplicable especial. Ahora bien, en tal caso deberán regir los principios de checks and balances entre poderes y de derechos adquiridos ${ }^{26}$.

El caso español ofrece un buen ejemplo de lo que se acaba de señalar: por una parte, se ha denominado "nueva normalidad" a la situación que rigió entre junio y octubre de 2020, sin que fuera una vuelta a la situación previa, dadas las circunstancias sanitarias, sociales y económicas. $Y$ por otra parte, la larga duración prevista de seis meses de la prórroga del tercer estado de alarma, aunque sea susceptible de reducción a cuatro si se producen determinados requisitos (solicitud por las CCAA ante nuevas circunstancias y se prevea la información parlamentaria por parte del Presidente del Gobierno -cada dos meses ante el Pleno del Congreso- y del Ministro de sanidad -cada mes ante la Comisión de sanidad del Congreso), plantea problemas de admisibilidad a la luz del criterio fijado: "la Comisión de Venecia ha declarado que "cuanto más dure el régimen de emergencia, más se alejará el Estado de los criterios objetivos que pueden haber validado el uso de las facultades de emergencia en primer lugar. Cuanto más tiempo persista la situación, menor será la

\footnotetext{
${ }^{24}$ Interim Report...CDL-AD(2020)018, párr. 46-49.

${ }^{25}$ Interim Report...CDL-AD(2020)018, párr. 25.

${ }^{26}$ Interim Report...CDL-AD(2020)018, párr. 26 y 27. 
justificación para tratar una situación como de carácter excepcional, con la consecuencia de que no puede abordarse mediante la aplicación de los instrumentos jurídicos normales""27.

El principio de necesidad afecta al tipo de normas que se aprueben durante el estado de emergencia. Se trata de normas que han de estar vinculadas con la situación de emergencia ${ }^{28}$. De este modo, no cabe aprovechar las normas dictadas para afrontar la emergencia para incluir normas estructurales con vocación de permanencia, como las que introducen cambios en la organización y funcionamiento de las instituciones ${ }^{29}$. Precisamente esto es lo que ha ocurrido en España en relación con ciertos cambios en la composición de la Comisión del CNI, aprobados durante la vigencia del primer estado de alarma de 2020.

Por último, y como consecuencia del principio de excepcionalidad, la Compilación recuerda que no se puede llevar a cabo una reforma constitucional bajo la vigencia de estados excepcionales ${ }^{30}$. Esto lo recogen muchas constituciones. En el caso español, respecto al inicio de la reforma (art. 169 $\mathrm{CE})$. Una cosa es que las constituciones se elaboren o se reformen de forma sustantiva como consecuencia de grandes crisis o cambios sociales o políticos (momentos constitucionales), y otra que tales cambios constitucionales tengan lugar en medio de la declaración de estados excepcionales.

\footnotetext{
${ }^{27}$ Interim Report...CDL-AD(2020)018, párr. 51. El párrafo reproduce un fragmento de CDL$A D$ (2016)037-e, Opinion on Emergency Decree Laws $N^{\circ}$ s 667-676 adopted following the failed coup of 15 July 2016, Opinion No. 865/2016, 12 December 2016, par 41, en la Compilación, pág. 22.

${ }_{28}^{28}$ Interim Report...CDL-AD(2020)018, párr. 23 y 24.

${ }^{29}$ CDL-AD(2016)037, Turkey - Opinion on Emergency Decree Laws Ns667-676 adopted following the failed coup of 15 July 2016, párr. 80, en la Compilación, pág. 22.

${ }^{30} \mathrm{CDL}-\mathrm{AD}(2017) 005$, Turkey - Opinion on the amendments to the Constitution adopted by the Grand National Assembly on 21 January 2017 and to be submitted to a National Referendum on 16 April 2017, párr. 29, en la Compilación, pág. 25, con cita, entre los ejemplos, a España. 
III. CRITERIOS DE LA COMISIÓN DE VENECIA EN RELACIÓN CON LA AFECTACIÓN A LOS DERECHOS Y A LA SEPARACIÓN DE PODERES DURANTE LOS ESTADOS DE EMERGENCIA

\section{Limitación y suspensión de derechos fundamentales}

Los derechos fundamentales pueden ser suspendidos, según recuerda la Compilación, "solo en circunstancias muy excepcionales" durante la vigencia de los estados excepcionales. Esta es la primera regla general a tener en cuenta. Distintos de la suspensión son la limitación o restricción de derechos contenida tanto en el Derecho internacional de los derechos humanos como en el Derecho constitucional de los derechos fundamentales, así como la derogación de las obligaciones derivadas de los tratados internacionales en materia de derechos humanos, prevista por algunos, como la Convención Europea de Derechos Humanos (art. 15) o el Pacto Internacional de Derechos Civiles y Políticos de la ONU (art. 4). Sin embargo, la nomenclatura utilizada en los distintos documentos de la Comisión de Venecia no siempre es homogénea: a veces se distingue suspensión y derogación, otras se emplean como sinónimos ${ }^{31}$. La Comisión analiza el régimen de afectación (sin distinguir ahora el tipo) de los estados de emergencia a los derechos tanto desde el punto de vista del Derecho internacional público como del Derecho constitucional de los Estados.

Como medida más radical del régimen de excepción en el campo de los derechos, la Comisión de Venecia alude a derogaciones temporales de las obligaciones derivadas de los tratados sobre derechos humanos para circunstancias excepcionales como "periodos de guerra o de otras emergencias públicas que amenacen la vida de la nación" (art. $15 \mathrm{CEDH}$ ). Esta cláusula ha sido invocada, durante la crisis del coronavirus, por varios Estados del Este de Europa (Estonia, Letonia y Rumanía). Tal derogación no puede ser total ${ }^{32}$, y está sujeta a los principios de necesidad, proporcionalidad y temporalidad así como a los requisitos formales de previa declaración del estado excepcional y

\footnotetext{
${ }^{31}$ En parte es un problema derivado de la traducción: en la versión inglesa y francesa de ambos documentos se utiliza el término derogación, mientras que en la traducción al español se opta por suspensión en el caso del art. 4 del PIDCyP y derogación en el art. 15 de la CEDH. La Convención Americana de Derechos Humanos opta por suspensión tanto en versión en español como en inglés (art. 27).

32 Interim Report...CDL-AD(2020)018, párr. 15-16.
} 
notificación a la organización internacional. Además, se recuerda que las autoridades de los Estados disponen de un amplio margen de apreciación a la hora de determinar la aplicación de los estados de emergencia, lo que, sin embargo, no hace desaparecer la "supervisión europea", en particular, del Tribunal Europeo de Derechos Humanos ${ }^{33}$.

Las constituciones suelen incluir también cláusulas de suspensión de derechos, que acotan la misma a situaciones de excepción, sean estados excepcionales o suspensiones individuales relacionadas con pertenencia a banda armada 0 terrorista (así el art. $55 \mathrm{CE}$ ). Se excluyen de la suspensión y la derogación varios derechos, en una lista de derechos que no siempre coincide en las distintas opiniones de la Comisión de Venecia, y, por ello mismo, no es una lista cerrada o exhaustiva. Hay un núcleo de derechos que aparece siempre en el Derecho internacional de los derechos humanos como derechos no objeto de derogación: el derecho a la vida, la prohibición de tortura y tratos inhumanos y degradantes, la prohibición de la esclavitud y el principio nullun crimen, nulla poena sine lege. Pero la Comisión de Venecia la considera una cláusula abierta, ya que dichos derechos son suspendibles "entre otros" 34 , lo que se traduce en la inclusión, en otras opiniones e informes, de la libertad de pensamiento, consciencia y religiosa ${ }^{35}$. Otras veces se amplía a la privacidad, libertad de expresión, derecho de voto, acceso a cargos públicos, reunión y asociación, además de otros principios básicos del Estado constitucional de Derecho ${ }^{36}$. Incluso se llega a hablar, en alguna ocasión, de derechos "implícitamente no derogables", como algunos derechos y garantías relacionados con la tutela judicial o procesales ${ }^{37}$.

De este modo, la Comisión de Venecia, como en general el Derecho internacional de los derechos humanos, adopta la técnica inversa que la

\footnotetext{
${ }^{33}$ CDL-AD(2016)037, Turkey - Opinion on Emergency Decree Laws Nº667-676 adopted following the failed coup of 15 July 2016, párr. 36, pág. 6, párr. 33, en la Compilación, pág. 10.

${ }^{34}$ Rule of Law Checklist, CDL-AD(2016)007 párr. 52, en la Compilación, pág. 4.

${ }^{35}$ CDL-AD(2016)006, Opinion on the Draft Constitutional Law on "Protection of the Nation" of France, párr. 28, en la Compilación, pág. 7. En las Reflexiones...CDL-PI(2020)005, párr. 58, la libertad religiosa queda relegada a una mención de la posible prohibición o limitación en las condiciones de celebración de "ceremonias religiosas". Ello choca con la amplitud de la protección del derecho fundamental a la libertad religiosa en documentos como el citado en esta misma nota.

${ }^{36}$ CDL-AD(2006)015, Opinion on the Protection of Human Rights in Emergency Situations, párr. 34, en la Compilación, pág. 10.

${ }^{37}$ CDL-AD(2016)037, Turkey - Opinion on Emergency Decree Laws Nos 667-676 adopted following the failed coup of 15 July 2016, párr. 159, en la Compilación, pág. 20. 
Constitución española respecto a la identificación de los derechos objeto de suspensión: mientras la Constitución señala los derechos que sí pueden ser suspendidos en el Estado de excepción y en el Estado de sitio, pero no en el de alarma, en lo que constituye un numerus clausus (art. 55.1 CE), los documentos internacionales identifican los que no pueden ser derogados en ningún caso, con mayor flexibilidad. Con una técnica delimitadora o con otra, queda identificado el núcleo básico de los derechos, objeto de protección prioritaria por el Derecho, y por ello, también durante los estados excepcionales. Se impone al respecto el principio de excepcionalidad o restrictivo, además del de legalidad o carácter expreso. Precisamente la aplicación del estado de alarma a la epidemia sanitaria ha conllevado medidas restrictivas de derechos que van más allá de la mera afectación, contemplada para tal estado excepcional. Se advierte un déficit regulador para situaciones de este tipo, que no encajan bien con ninguno de los estados excepcionales previstos ni tampoco con la legislación de emergencias sanitarias (material y la garantía contenciosa de la misma).

Por último, la doctrina de la Comisión de Venecia se refiere a otros derechos que pueden ser objeto de limitación o restricción pero no de suspensión. En tal caso se aplicarían los principios (o test, en la otra denominación empleada) de necesidad y proporcionalidad ${ }^{38}$, además del de legalidad y de fin legítimo ${ }^{39}$, como ya hemos avanzado. La seguridad nacional, la salud pública o la integridad territorial son bienes jurídicos públicos a proteger "en una sociedad democrática", tal y como lo contempla el Convenio Europeo de Derechos Humanos para varios derechos reconocidos (por ej. art. 10), y que justifican esas posibles restricciones a los derechos.

La Comisión de Venecia constata como la crisis de la covid-19 ha mostrado la relevancia de la protección de los derechos sociales y el desarrollo de servicios públicos sanitarios de carácter universal, con la posible invocación de la Carta Social Europea para fundar respuestas a demandas ${ }^{40}$. Ello muestra una preocupación social e intervencionista, como respuesta a las necesidades de los tiempos de grave crisis social y económica, que va más allá de la protección estricta de los derechos fundamentales civiles y políticos, que están en el

\footnotetext{
${ }^{38}$ Ibidem.

39 Reflexiones...CDL-PI(2020)005, párr. 40. Todos ellos también en Interim Report...CDLAD(2020)018, párr. 14.

${ }^{40}$ Interim Report...CDL-AD(2020)018, párr. 11. 
Convenio Europeo de Derechos Humanos. Ello sin embargo no supone ni aprovechar la coyuntura para eliminar ni para limitar desproporcionadamente tales derechos fundamentales, y deja en las manos de cada Estado la concreción de los medios para alcanzar el fin.

Además de las garantías judiciales de los derechos, a la que se aludirá posteriormente, la Comisión se refiere a la "contribución crucial" que puede desempeñar el Ombudsman en la protección de los derechos durante la vigencia de los estados de emergencia y la asistencia que puede prestar a los ciudadanos afectados por las medidas adoptadas ${ }^{41}$.

Más allá de los derechos fundamentales y las tareas del Estado social, las Reflexiones indican que la declaración, activación y aplicación de los poderes de emergencia pueden implicar suspensiones en la aplicación del derecho civil, laboral, de la Seguridad Social o financiero. Ello incluye que los gobiernos puedan asumir temporalmente "subsidios a negocios" o relajar controles. Ahora bien, dichas suspensiones del derecho ordinario deben hacerse a través de leyes especiales y mantener así su carácter transitorio ${ }^{42}$.

\section{Especial atención al derecho de sufragio y la celebración de elecciones}

Particular atención ha merecido, para la Comisión de Venecia, el derecho de sufragio y, en concreto, la celebración de elecciones, especialmente en el Interim Report. Ello se justifica por su atención especial a los asuntos electorales y la arraigada experiencia en supervisión de procesos electorales. La Comisión admite que no hay un criterio claro acerca de la celebración o aplazamiento de elecciones durante la situación de emergencia. Una menor participación en tales circunstancias, se admite, supondría una distorsión en el ejercicio de la democracia.

El criterio que se sigue en relación con los referendos es más unívoco: la Comisión apuesta "en principio" por su aplazamiento, sobre todo si es de iniciativa gubernativa ${ }^{43}$. Ello no obstante, hemos asistido recientemente a los

\footnotetext{
${ }^{41}$ Interim Report...CDL-AD(2020)018, párr. 99.

${ }^{42}$ Reflexiones...CDL-PI(2020)005, párr. 53-57.

43 Interim Report...CDL-AD(2020)018, párr. 115. 
referéndums constitucionales de Italia y de Chile, además de Suiza, en los que la participación habida no aporta datos concluyentes en términos generales.

En relación con las elecciones, la Comisión de Venecia pondera las ventajas e inconvenientes de su celebración a la luz de dos de los principios del derecho de sufragio: por un lado, la preservación del carácter libre del voto invita al aplazamiento, para que pueda desarrollarse la campaña y la jornada electoral con todas las garantías y con la máxima participación y, por otro lado, el carácter periódico del voto es un argumento en favor de la celebración cuando corresponda $^{44}$. Ahora bien, en caso de celebrarse las elecciones, se debe garantizar el pleno ejercicio de los derechos políticos, así como los estándares internacionales sobre elecciones libres y limpias ${ }^{45}$. En tal caso, se menciona el ejercicio del derecho de sufragio a través de varias modalidades: voto por correo (se cita como ejemplos de la multiplicación de dicho tipo de voto en los casos de las elecciones a los Parlamentos de Galicia y el País Vasco) o voto electrónico, si cumple con las garantías debidas ${ }^{46}$.

La Comisión de Venecia se pronuncia también sobre quién debe tomar la decisión sobre el aplazamiento electoral. Este, si no está contemplado en la Constitución o en una ley orgánica (lo cual por lo general no suele suceder), debe al menos acordarlo el propio Parlamento, con exigencia de mayoría amplia (sobre todo si es un aplazamiento superior a 6 meses) o el Gobierno o la Administración electoral (en caso de que sea por un periodo de menos de dos meses) ${ }^{47}$. Queda en medio una zona gris que invita a valorar los diferentes bienes en juego.

Al hacerse mención de los Estados que permiten aplazar elecciones en caso de declaración de los estados excepcionales, la Compilación cita a España, y a continuación hace mención a los Estados que no permiten la disolución del Parlamento durante la vigencia de los Estados excepcionales, con referencia

\footnotetext{
${ }^{44}$ Interim Report...CDL-AD(2020)018, párr. 102-103.

${ }^{45} \mathrm{CDL}-\mathrm{AD}(2017) 005$, Turkey - Opinion on the amendments to the Constitution adopted by the Grand National Assembly on 21 January 2017 and to be submitted to a National Referendum on 16 April 2017, párr. 32, en la Compilación, pág. 28.

${ }^{46}$ Interim Report...CDL-AD(2020)018, párr. 134-135.

${ }^{47}$ Reflexiones...CDL-PI(2020)005, párr. 118. Se repite en Interim Report...CDL-AD(2020)018, párr. 123.
} 
otra vez a nuestro país ${ }^{48}$. Esto último es justamente lo que indica el art. 116.5 $\mathrm{CE}$; lo primero, en cambio, es correcto si se trata de una consecuencia de no poder disolver las Cortes Generales, pero si estas ya están disueltas en el momento de la Declaración (como ocurrió en los Parlamentos autonómicos de Galicia y el País Vasco el pasado abril de 2020), existe un vacío normativo, que debería ser colmado cuanto antes, para evitar soluciones sobre la marcha discutibles, como las que se adoptaron en ambas Comunidades Autónomas y que permitieron celebrar sendas elecciones en julio de 2020, por decreto del respectivo presidente de la Comunidad Autónoma, con el acuerdo de los partidos presentes en las Asambleas, y con el visto bueno de la Administración electoral. En cualquier caso, como tiene dicho la Comisión de Venecia, la reforma de la ley electoral debe realizarse al menos con un año de antelación respecto a la convocatoria de que se trate, salvo si se trata de cuestiones técnicas o de detalle ${ }^{49}$. Además, es bueno que la reforma tenga carácter general y no se apruebe para el caso concreto.

\section{Redistribución de los poderes durante la emergencia}

Respecto a la redistribución y ejercicio de los poderes durante la vigencia de los estados de emergencia, la Comisión de Venecia parte de la afectación y cambio frecuente que supone su declaración sobre la distribución de poderes en Derecho comparado ${ }^{50}$. Como se ha avanzado, la Comisión de Venecia se refiere a los principios de control y de leal cooperación entre instituciones. Ambos principios constituyen las dos caras de la relación entre instituciones, nacionales y territoriales, y entre mayoría y oposición en la declaración y aplicación del estado de emergencia. Así pues, se ha de intentar el más amplio consenso político posible en sede parlamentaria en la adopción del estado de emergencia y, al mismo tiempo, el control parlamentario en su ejecución, además de la cooperación interterritorial en los Estados políticamente compuestos. Bajo este prisma cabe interpretar el significado del principio de normal funcionamiento de los poderes públicos; no como equivalente a una normalidad que precisamente la emergencia deja de lado. La Compilación lleva

\footnotetext{
${ }^{48}$ Párrafo 31 de la CDL-AD(2017)005, Turkey - Opinion on the amendments to the Constitution adopted by the Grand National Assembly on 21 January 2017 and to be submitted to a National Referendum on 16 April 2017, en la Compilación, pág. 31.

${ }^{49}$ Code of Good Practices in Electoral Matters. Guidelines and Explanatory Report, CDL-AD (2002) 23, II.2.

${ }^{50}$ Interim Report...CDL-AD(2020)018, párr. 52. 
a cabo una referencia a España, en concreto, al art. 116.5 CE, como ejemplo de garantía del principio de normal funcionamiento de los poderes públicos: "El estado de emergencia puede o no implicar cambios en la distribución de poderes entre los órganos del Estado o cambios en las competencias de dichos órganos. En algunos casos (por ejemplo, en España y Portugal) el funcionamiento normal de los órganos constitucionales no se ve afectado por el estado de emergencia. [...] Normalmente, la declaración del estado de emergencia implica la transferencia de poderes adicionales al ejecutivo." ${ }^{21}$

La Comisión de Venecia parte de una doble premisa de tipo fáctico en sus consideraciones sobre el tema. La primera es sobreentendida: la constatación de que la crisis sanitaria ha incidido en el funcionamiento normal de la vida parlamentaria (dificultad de mantener reuniones presenciales con la asistencia de todos los parlamentarios). La segunda, el refuerzo en la posición de los gobiernos centrales, mientras que los Parlamentos han quedado "relegados a un rol secundario" 52 . Ante ello, la Comisión aboga por enfatizar los poderes del Parlamento de control sobre el Gobierno, así como por la necesidad de mayorías cualificadas en los Parlamentos para aprobar la declaración y/o su prórroga. Esto último persigue el objetivo de sumar a la oposición en la decisión. En efecto, "[m]uchas constituciones prevén la posibilidad de que el ejecutivo legisle en situaciones de emergencia. El Parlamento debe participar en este proceso mediante la aprobación de la declaración del estado de emergencia, y/o mediante el examen a posteriori de los decretos de emergencia o cualquier prórroga del período de emergencia. La participación de la oposición en esos asuntos puede garantizarse exigiendo una mayoría cualificada para la prolongación del estado de emergencia más allá del período original (CDL$A D(2016) 006$, párrafo 1). 63). También puede ser útil limitar los poderes legislativos del ejecutivo en situaciones de emergencia a determinadas cuestiones específicas, de modo que el ejecutivo no pueda utilizar sus funciones legislativas para suprimir los derechos de la oposición. La Comisión de Venecia ha subrayado que la vida parlamentaria debe continuar durante todo el estado de emergencia (...) Se recomienda no emprender enmiendas constitucionales durante situaciones de emergencia (CDL-AD(2017)005,

\footnotetext{
${ }^{51}$ CDL-STD(1995)012, Emergency Powers, en la Compilación, pág. 14.

52 Interim Report...CDL-AD(2020)018, párr. 61 y 64. 
párrafo 29). Estas limitaciones impiden que el ejecutivo utilice la emergencia como pretexto para restringir los derechos de la oposición. ${ }^{53}$.

Según se indica en las Reflexiones, al Parlamento corresponde la aprobación del estado de emergencia, o al menos su prórroga. Esta última es la previsión en España respecto al estado de alarma (art. $116 \mathrm{CE}$ ). Y ofrece un criterio al legislador: para aprobar la prórroga de la vigencia del estado excepcional se "puede" exigir una mayoría cualificada ${ }^{54}$. Se alude igualmente a la posible creación de comisiones de investigación, para facilitar el control sobre el uso gubernamental de los poderes de excepción ${ }^{55}$. De este modo, se concreta el principio de protección de la oposición, sea con exigencias de consenso parlamentario para la declaración o al menos para el mantenimiento de los poderes de excepción, de modo que no se utilicen estos para obviar al Parlamento o para limitar las facultades de las minorías parlamentarias, sea en el control del ejercicio de los poderes excepcionales por parte del Gobierno.

La Comisión de Venecia hace referencia a la utilización frecuente en tales circunstancias excepcionales de normas gubernamentales con rango de ley, como los decretos ley, y a la intervención del Parlamento en su convalidación (en España más de treinta desde marzo 2020, además de los numerosos autonómicos). Las Reflexiones resaltan el uso legítimo de dichos tipos de normas durante los estados excepcionales, pero con límites estrictos de vigencia y objeto ${ }^{56}$.

Las circunstancias particulares provocadas por la crisis sanitaria -reducción de la movilidad y reuniones públicas- obliga a cambios en la forma de funcionar del Parlamento. A ello dedica el último documento de la Comisión de Venecia, el Interim Report, algunas consideraciones sobre cómo han afrontado la situación los Estados miembros de la UE: trabajo remoto, refuerzo de instrumentos digitales, etc. La advertencia principal de la Comisión al respecto se centra en la necesidad de mantenimiento de sesiones plenarias y el no

\footnotetext{
${ }^{53}$ CDL-AD(2019)015, Parameters on the Relationship between the Parliamentary Majority and the Opposition in a Democracy: a checklist, párr. 121, en la Compilación, pág 16.

${ }^{54}$ Reflexiones...CDL-PI(2020)005, párr. 84.

${ }^{55}$ Reflexiones...CDL-PI(2020)005, párr. 82.

${ }^{56}$ Reflexiones...CDL-PI(2020)005, párr. 63 y 64, con cita del CDL-AD(2019)019, Parameters On The Relationship Between The Parliamentary Majority And The Opposition In A Democracy: A Checklist, párr. 119-121. Los datos de situación en Interim Report...CDL-AD(2020)018, párr. 64. 
reemplazo temporal de parlamentarios ni la reducción de la asistencia a las sesiones, puesto que la discusión cara a cara es crucial para el debate ${ }^{57}$.

En definitiva, la Comisión de Venecia aplica a este tema su doctrina reiterada acerca de la centralidad del Parlamento en la vida pública de un país. Un rol secundario del Parlamento durante el estado de emergencia puede afectar al funcionamiento de la democracia, de ahí que subraye las funciones que ha de ejercer este en relación con dicho estado. Por ello, concluye la Comisión de Venecia: "la crisis de covid-19 no debe aprovecharse para hacer más poderosos a los gobiernos a expensas de los parlamentos y, en todo caso, no de manera permanente. A fin de prepararse para el futuro, debería considerarse seriamente la posibilidad de determinar el mejor escenario y asegurar que se disponga del marco regulatorio necesario para cumplirlo, así como determinar en qué grado algunas de esas medidas podrían mantenerse a lo largo del tiempo, independientemente de que exista o no una emergencia. El Parlamento debe ser el centro de la vida política de un país y, para mantener esta condición, deben existir los instrumentos y mecanismos necesarios para garantizarlo. La continuación de la labor del Parlamento debe considerarse un requisito esencial durante una crisis, y deben adoptarse medidas -por ejemplo, permitir y mejorar las reuniones digitales del Parlamento cuando las reuniones físicas sean imposibles- para mantener la labor parlamentaria sin dificultades durante tales situaciones en el futuro." 58 .

Las Reflexiones aluden también al papel de los expertos ${ }^{59}$ y del ejército. Respecto de las fuerzas armadas, la Compilación recoge el ejemplo de España para aludir a la existencia en algunos países de unidades militares especiales para desempeñar tareas durante la emergencia (la UME $)^{60}$. La aportación de los expertos o técnicos y de los órganos consultivos apenas es desarrollada, salvo para indicar que contribuye a reforzar al Gobierno y a debilitar al Parlamento. En todo caso, el denominado "acercamiento tecnocrático" nos pone ante la cuestión relevante de la relación entre ciencia y toma de

\footnotetext{
${ }^{57}$ Interim Report...CDL-AD(2020)018, párr. 75. Con cita en nota a pie de un trabajo de Piedad García-Escudero, de 2010.

${ }^{58}$ Interim Report...CDL-AD(2020)018, párr. 72.

${ }^{59}$ Reflexiones...CDL-PI(2020)005, párr. 69.

${ }^{60}$ CDL-AD(2008)004, Report on the Democratic Control of the Armed Forces, párr. 125, en la Compilación, pág. 27
} 
decisiones públicas en situaciones de crisis y ante cómo se produce la gestión del riesgo y la asunción de responsabilidades ${ }^{61}$.

Por último, también la organización territorial del Estado en sistemas descentralizados se ve afectada por los estados de emergencia. Así, en lo que respecta a la relación entre el Gobierno central y los territoriales, la Comisión de Venecia admite, dentro de la escasa atención dedicada al asunto, como criterio general, que los poderes del Gobierno central puedan limitar los de las autoridades subnacionales: "En algunos Estados federales, la declaración del estado de emergencia puede suponer el traspaso de competencias de las autoridades estatales y locales al Gobierno central."62. En otros documentos se limita el alcance de dicha limitación, que no podría llegar a la suspensión de la autonomía $^{63}$, ni a que la recentralización vaya más allá de las exigencias del estado excepcional ${ }^{64}$. Las Reflexiones se muestran más cautelosas y subrayan la aplicación de los principios de leal colaboración y de respeto mutuo ${ }^{65}$. También en el plano territorial se da un mayor protagonismo de los Ejecutivos sobre los Legislativos, ya sea a través de la aprobación de decretos-ley, ya de decretos y demás normas infralegales para hacer frente a la emergencia.

\section{Control judicial y mantenimiento del Estado de Derecho}

La Comisión de Venecia reitera la necesidad del control judicial, además del parlamentario, sobre la declaración del estado de emergencia y respecto a las medidas adoptadas durante su vigencia por el Ejecutivo "contra los riesgos de abuso". Ello está vinculado con el principio de mantenimiento del Estado de Derecho. El margen de discreción mayor que se reconoce al gobierno no empece que el sistema judicial no deba proveer a los individuos de un "recurso efectivo" en caso de lesión de los derechos ${ }^{66}$. Dicho control puede corresponder tanto a la jurisdicción ordinaria como a la constitucional de cada

\footnotetext{
${ }^{61}$ Sobre ello es ineludible remitirse a José ESTEVE PARDO, "La apelación a la ciencia en el gobierno y gestión de la crisis de la Covid-19", Revista de Derecho Público: teoría y método, vol 2, 2020, págs. 35-50.

${ }^{62}$ CDL-STD(1995)012, Emergency Powers, en la Compilación, pág. 26. Se repite en Interim Report...CDL-AD(2020)018, párr. 54.

${ }^{63} \mathrm{CDL}-\mathrm{AD}(2011) 049$, Opinion on the draft law on the legal regime of the state of emergency of Armenia, párr. 34, en la Compilación, pág. 26.

${ }^{64}$ CDL-AD(2017)021, Turkey - Opinion on the Provisions of the Emergency Decree-Law N ${ }^{\circ} 674$ of 1 September 2016 which concern the exercise of Local Democracy, párr. 92, en la Compilación, pág. 26.

${ }^{65}$ Reflexiones...CDL-PI(2020)005, párr. 61.

${ }^{66}$ Interim Report...CDL-AD(2020)018, párr. 77 y 79.
} 
Estado, además de la que puedan ejercer órganos internacionales, sea judiciales o cuasi judiciales, en particular, el Tribunal Europeo de Derechos Humanos. Para el cumplimiento de este principio de control, se recalca la garantía de independencia de los tribunales y el mantenimiento del funcionamiento de los mismos, salvo casos de absoluta necesidad o de imposibilidad material ${ }^{67}$.

En los documentos de la Comisión se advierte una preferencia por la justicia constitucional. $\mathrm{E}$, incluso, se añade que esta deba tener el poder de ordenar "medidas provisionales" ${ }^{8}$. Dependerá de la legislación de cada Estado la delimitación de funciones entre jurisdicción ordinaria y constitucional. La preferencia por la justicia constitucional tiene sentido al menos para la declaración del estado de emergencia, aunque tal opción puede afectar negativamente a la posibilidad misma de reclamar el control. En el caso español, es sabido que la legitimación para el control de constitucionalidad es restringida. Esta cuestión está en la base de la consideración, en España, del decreto de declaración del estado de alarma y de los de prórroga como actos con valor de ley (STC 83/2016, en relación con el primer estado de alarma de 2010). Ello conlleva la imposibilidad de impugnación de las normas que declaran o prorrogan el estado de alarma por parte de particulares afectados o interesados. Otra cosa son los actos o decretos de aplicación, cuyo control a posteriori corresponde, en primer lugar, a la jurisdicción contenciosoadministrativa, y, subsidiariamente, a la revisión del Tribunal Constitucional a través del recurso de amparo por lesión de un derecho fundamental. A parte de lo anterior, está la previsión de autorización o ratificación judicial de las medidas administrativas que quedan al margen de la cobertura del decreto de alarma, como se ha visto (Ley de la jurisdicción contencioso-administrativa).

Sin embargo, la Comisión de Venecia admite que el control jurisdiccional suela limitarse en la práctica debido a un "self restrain judicial", como ha ocurrido ya en ciertos pronunciamientos sobre la declaración del estado de emergencia por la pandemia o bien las medidas adoptadas en su aplicación (Corte Constitucional de Chequia o de Serbia; diferente ha sido el caso de Portugal o Francia). Ello es particularmente notorio en relación con las derogaciones

${ }^{67}$ Reflexiones...CDL-PI(2020)005, párr. 87 y 89.

68 Reflexiones...CDL-PI(2020)005, párr. 88. Se matiza la preferencia por la justicia constitucional, pero no la adopción por esta de medidas provisionales, en el Interim Report...CDL-AD(2020)018, párr. 78. 
previstas en los instrumentos internacionales de derechos humanos, donde el Tribunal Europeo de Derechos Humanos ha reconocido un amplio margen de apreciación a cada Estado ${ }^{69}$. De todos modos, ello no significa renunciar a todo control en tal caso ni en general. Los principios de necesidad y proporcionalidad se aplican para valorar la restricción y derogación de derechos.

En la práctica, y en el caso español, se observa que dicha deferencia judicial con el Gobierno ha sido habitual -aunque ciertamente no unánime- en los distintos tribunales con relación a la aplicación de las medidas restrictivas de derechos fundamentales en la gestión de la epidemia, además de la que el Tribunal Constitucional ha mostrado habitualmente respecto al control de los decretos ley (y más los aprobados durante la anterior crisis financiera). Por ahora, el Tribunal Constitucional no se ha pronunciado aún sobre el recurso de inconstitucionalidad interpuesto por los diputados de Vox contra el primer decreto del estado de alarma de marzo 2020; el mismo grupo parlamentario ha interpuesto otro contra el tercero en noviembre 2020.

\section{CONCLUSIONES}

La lógica que suele predominar en la racionalización del uso de los poderes excepcionales en los Estados constitucionales de Derecho ha sido hasta ahora la propia de los estados excepcionales derivados de crisis políticas graves de orden público o de crisis naturales o sanitarias de efectos circunscritos. De ahí las dificultades que plantea la inclusión de emergencias como la que ahora nos asola que, en parte -aunque solo en parte- explica las variadas opciones seguidas en los Estados europeos. Esa misma lógica ha sido la orientación constante en los estudios jurídicos sobre los estados excepcionales hasta la fecha en los distintos Estados, incluida España. Pero lo anterior no significa que muchos de los criterios establecidos legal o jurisdiccionalmente no sean aplicables a la actual pandemia, aunque se produzcan ciertos vacíos normativos y se requieran ciertas adaptaciones. Ello, a buen seguro, obligará a los legisladores a contemplar regulaciones específicas que den cobertura adecuada a situaciones de este género.

\footnotetext{
${ }^{69}$ Interim Report...CDL-AD(2020)018, párr. 21 y 83-84, con cita de doctrina del TEDH. 
Lo que se acaba de decir en términos generales se aplica también a los documentos relacionados con los estados de emergencia adoptados por la Comisión de Venecia en los años anteriores. Ello explica la rápida y ágil reacción de la Comisión de Venecia desde el inicio de la pandemia. Los dos últimos documentos que se han analizado en estas páginas, las Reflexiones y el Interim Report, adaptan los estándares generales de los estados de emergencia (recopilados en la Compilación) a las particularidades de la pandemia presente, y vienen a rellenar este vacío en los documentos de órganos internacionales. Queda pendiente el seguimiento de lo que suceda a partir de octubre de 2020, en lo que se ha denominado "segunda ola" de la pandemia, en la que nos encontramos cuando se escriben estas líneas.

De dichas aportaciones, basadas en el Derecho internacional, el Derecho constitucional comparado y las buenas prácticas habidas en los Estados, se derivan criterios que pueden servir para orientar reformas legislativas o para valorar e interpretar las normas vigentes. Por ello tales documentos merecen una lectura atenta.

La Comisión de Venecia constata como el estado de emergencia supone tanto una inaplicación de las normas ordinarias sobre derechos como cambios en los poderes de las diferentes instituciones estatales. De ahí, como se ha visto, que subraye el carácter temporalmente limitado o provisional y "verdaderamente" excepcional de las medidas que se adopten, preferiblemente aplicando las normas constitucionales de excepción, y su sometimiento a los principios de necesidad y proporcionalidad. No obstante, ello no empece que el Estado de Derecho siga plenamente vigente y que se prevean garantías frente a posibles malos usos en la adopción y aplicación de dichas medidas por los gobiernos ${ }^{70}$. Sin embargo, la crisis del coronavirus ha puesto de manifiesto, de entrada, el recurso a instrumentos normativos distintos de los estados de excepción en no pocos países. Incluso en los que los han aplicado, se ha hecho de forma simultánea a la aprobación de decretos ley, y tras el cese de dichos estados, no se ha vuelto a la normalidad constitucional anterior, sino que se han dictado o aplicado normas especiales bajo el paraguas de una "nueva normalidad", al perdurar la crisis sanitaria y cobrar fuerza la crisis económica y social. De este modo, sea por la larga duración de los estados de emergencia, o bien por la

\footnotetext{
${ }^{70}$ CDL-AD(2016)006, Opinion on the Draft Constitutional Law on "Protection of the Nation" of France, párr. 51, en la Compilación, pág. 12. 
utilización de legislación de emergencia fuera de dichos estados, nos hallamos ante la aplicación por largo tiempo de normas especiales, de carácter en principio transitorio, generalmente de jerarquía infralegal (órdenes ministeriales y resoluciones), que amplían el alcance del derecho de emergencia más allá de sus confines clásicos. Pero ello entraña que queden difuminadas las garantías especiales propias del derecho de excepción. Esto sucede particularmente con el control parlamentario de los actos del Ejecutivo.

Como resumen de la posición de la Comisión de Venecia respecto a los estados de emergencia, podemos concluir reproduciendo un fragmento relevante de uno de sus documentos anteriores a esta crisis sanitaria, pero de plena valía en la actual. En él queda clara la opción de dicho órgano consultivo internacional por el modelo constitucional frente al soberano o decisionista de estado excepcional y lo que ello entraña: la garantía de la seguridad del Estado y la seguridad pública en una democracia con pleno respeto del Estado de Derecho y los derechos fundamentales, que son los objetivos fundacionales del Consejo de Europa y de la Comisión de Venecia. La cita es larga, pero merece la pena como recordatorio de lo que siempre resulta oportuno tener presente al aplicar el derecho de emergencia: "La seguridad del Estado y de sus instituciones democráticas, así como la seguridad de sus funcionarios y de la población, son intereses públicos y privados vitales que merecen protección y pueden conducir a una suspensión temporal de ciertos derechos humanos y a una división extraordinaria de poderes. Sin embargo, los gobiernos autoritarios han abusado de los poderes de emergencia para mantenerse en el poder, silenciar a la oposición y restringir los derechos humanos en general. Por lo tanto, es esencial establecer límites estrictos a la duración, las circunstancias y el alcance de esos poderes. La seguridad del Estado y la seguridad pública sólo pueden garantizarse eficazmente en una democracia que respete plenamente el Estado de derecho. Ello requiere un control parlamentario y una revisión judicial de la existencia y duración de una situación de emergencia declarada para evitar abusos."71

\footnotetext{
${ }^{71}$ Rule of Law Checklist (CDL-AD(2016)007), párr. 51, en la Compilación, pág. 4. 